\title{
Biodiversity and spatial distribution of epiphytic ferns on Alsophila setosa Kaulf. (Cyatheaceae) caudices in Rio Grande do Sul, Brazil
}

\author{
Schmitt, JL. ${ }^{\mathrm{a}, \mathrm{b} *}$ and Windisch, $P G{ }^{\mathrm{a}}$ \\ aPrograma de Pós-Graduação em Botânica, Universidade Federal do Rio Grande do Sul - UFRGS, \\ Av. Bento Gonçalves, 9500, CEP 91501-970, Porto Alegre, RS, Brazil \\ 'Programa de Pós-Graduação em Qualidade Ambiental, Universidade Feevale, \\ RS-239 2755, CEP 93352-000, Novo Hamburgo, RS, Brazil \\ *e-mail: jairols@feevale.br \\ Received March 11, 2009 - Accepted July 16, 2009 - Distributed August 31, 2010
}

(With 2 figures)

\begin{abstract}
The extractive exploitation of the tree fern Alsophila setosa Kaulf. alters forest formations and diminishes the availability of micro-habitat for epiphytes. A survey of epiphytic fern communities on A. setosa at 16 study sites in different forest formations in the State of Rio Grande do Sul, Brazil, documented the occurrence of 31 species representing 16 genera and six families. The greatest richness of species occurred in Polypodiaceae (39\%) and in the genus Asplenium L. (22\%). Habitual holoepiphyte was the predominant ecological category, representing $61 \%$ of the species. Similarity analysis demonstrated heterogeneity in the composition of the epiphytic ferns at the study sites and indicated that the vegetation type is not the main factor for floristic difference. The lowest total specific richness (5) was recorded for the seasonal deciduous forest site. The majority of the sites presented similar averages of phorophyte height and epiphyte richness per caudex. In $25 \%$ of the sites the height of the host plants presented significant correlation with specific richness. Considering that the majority of the epiphytes are habitual and that some of them occur exclusively or preferentially on tree ferns, the maintenance of these host plants in the vegetation is essential for the conservation of epiphytic species.
\end{abstract}

Keywords: composition, species richness, epiphytism, tree fern, conservation.

\section{Biodiversidade e distribuição espacial de filicíneas epifíticas sobre cáudices de Alsophila setosa Kaulf. (Cyatheaceae) no Rio Grande do Sul, Brasil}

\begin{abstract}
Resumo
A exploração extrativista da samambaia arborescente Alsophila setosa Kaulf. altera as formações florestais e diminui a disponibilidade de microhabitats para epífitos. Um levantamento das comunidades de filicíneas epifíticas sobre A. setosa em 16 sítios de diferentes formações florestais, no Estado do Rio Grande do Sul, Brasil, documentou a ocorrência de 31 espécies representando 16 gêneros e seis famílias. A maior riqueza de espécies ocorreu em Polypodiaceae (39\%) e no gênero Asplenium L. (22\%). A categoria ecológica predominante foi a de holoepífito habitual, representando $61 \%$ das espécies. A análise de similaridade evidenciou heterogeneidade na composição de filicíneas epifíticas dos sítios estudados e indicou que o tipo vegetacional não é o principal fator de diferenciação florística. A menor riqueza específica total (5) foi registrada em sítio de floresta estacional decidual. A maioria dos sítios apresentou médias similares de altura dos forófitos e de riqueza de epífitos por cáudice. Em 25\% dos sítios, a altura das plantas hospedeiras apresentou correlação significativa com riqueza específica. Considerando que a maioria dos epífitos é habitual e que alguns deles ocorrem exclusivamente ou preferencialmente sobre samambaias arborescentes, a manutenção dessas plantas hospedeiras, na vegetação, é essencial para a conservação de espécies epifíticas.
\end{abstract}

Palavras-chave: composição, riqueza de espécies, epifitismo, samambaia arborescente, conservação.

\section{Introduction}

Epiphytes constitute an important, yet little known, component of the biodiversity of tropical forests (Mucunguzi, 2007). Ferns compose a group of plants worthy of attention in the epiphytic environment, considering that about 2,600 species around the world are epiphytic (Kress, 1986). Among adaptive strategies that favour epiphytism in those plants, which can operate in several combinations, are: low light saturation point; poikilohydry; frond trichomes; wind dispersal of spores; high tolerance for instability of substrate nutrients (Müller et al., 1981; Benzing, 1987, 1990; Page, 2002); fine to filiform, long and ramified rhizome, besides reduced radicular system and small fronds (Dubuisson et al., 2003).

Tree ferns are host species that create a surface which acts as mechanical support to a diverse epiphytic flora, including bryophytes and vascular plants (lycophytes, ferns 
and angiosperms), some of them growing exclusively or preferentially on this substratum (Sehnem, 1977; Cortez, 2001; Windisch, 2002; Moran et al., 2003; Roberts et al., 2005; Schmitt and Windisch, 2005 ). However, tree ferns are subject to commercial exploitation, decreasing the availability of microhabitats for epiphytic species.

Among the tree ferns used for decoration and landscape gardening in the State of Rio Grande do Sul, Brazil, Alsophila setosa is currently being extracted from primary and secondary forests (Windisch, 2002). Populations are also being reduced by deforestation for lumbering and for the use of land for grazing or agriculture (Schmitt and Windisch, 2005). The plants grow up to ca. $10 \mathrm{~m}$ tall (Schmitt and Windisch, 2005) have a caudex with scars (from fallen fronds) on the basal portion and remains of stipe bases (with blackish spines) on the distal part. The growth of the plants seems to be limited by the forest canopy. In adult plants, the crown is up to $3 \mathrm{~m}$ long and has fronds with tripinnate-pinnatisect laminae (Schmitt and Windisch, 2006).

The study of plant communities is essentially comparative and the physiognomy of a vegetation stand is the result of its composition in terms of plant species or types (Focht and Pillar, 2003). The richness and composition of the epiphytic flora is influenced by the phorophyte (Heatwole, 1993; Medeiros et al., 1993; Benzing, 1995; Rudolph et al., 1998; Nieder et al., 1999; Roberts et al., 2005; Schmitt et al., 2005; Fraga et al., 2008), as well as by the vegetation and climate within the forest (Gentry and Dodson, 1987; Nieder et al., 1997). Alsophila setosa grows in pioneer formations, dense humid forests, mixed humid forests with Araucaria angustifolia, seasonal semideciduous forests and seasonal deciduous forests in South and Southeastern Brazil, between 20 and 1,800 m elevation. This study analyses the species richness and floristic composition of epiphytic ferns on A. setosa in different types of vegetation in the State of Rio Grande do Sul (Southern Brazil); the similarity of these epiphytic communities among different sites; the influence of caudex height on species richness and compares the species richness among distinct sites.

\section{Material and Methods}

\subsection{Study area}

Floristic data of Alsophila setosa epiphytes were obtained from 16 sites (Figure 1) in the State of Rio Grande do Sul (Southern Brazil) as listed in Table 1. The climate is humid-temperate, with rainfall throughout the year (Moreno, 1961). Average yearly temperatures range from 14 to $20{ }^{\circ} \mathrm{C}$, with the coolest months from 10 to $15^{\circ} \mathrm{C}$, with frost occurring frequently in the highest altitudes (Quadros and Pillar, 2002).

\subsection{Locations and main environmental variables of study sites}

The geographical coordinates and altitude were acquired by Global Positioning System (GPS). The annual precipitation data was obtained from the Climatic Information Bulletin, available on the database from the National Civil Defense Secretariat. The vegetational types followed the classification presented by the RADAMBRASIL Project (Teixeira et al., 1986), recognising the following types of forest: seasonal deciduous, seasonal semideciduous, mixed humid, dense humid, as well as pioneer formation (Table 1).

\subsection{Floristic composition}

At each site, ca. $20,000 \mathrm{~m}^{2}$ of forest area was surveyed to establish the species of epiphytic ferns on Alsophila setosa. The survey of the caudex surface was performed by direct observation of the plants or with the help of binoculars. The botanical classification followed the system proposed by Smith et al., (2006) and taxonomic identification was based on the specialised literature. Voucher specimens were deposited at the Herbarium Anchieta (PACA), at Universidade Vale do Rio dos Sinos, São Leopoldo - RS (Brazil). Presence-absence data of epiphytic species were subjected to Palaeontological Statistics - PAST Program in order to determine the floristic similarity among sites (by Ward's method for cluster analysis). In Ward's method, a Euclidean distance measure is the inherent algorithm and clusters are joined so that the within-group variance is minimised (Hammer et al., 2003).

\subsection{Ecological categories}

The epiphytes were classified according to the phorophyte, using the categories proposed by Benzing (1990): habitual holoepiphytes (mostly present in the epiphytic habitat), facultative (occurring in the epiphytic habitat as well as terrestrial plants), accidental (preferentially terrestrial) and secondary hemiepiphytes (germinates on/in soil but after contact with the phorophyte the basal part with radicular/ caulinar portions degenerates).

\subsection{Phorophyte height and species richness}

At each study site, 10 caudices were randomly selected among those with epiphytes (based on the previous records for floristic composition). The average values of species richness and caudex height were compared from distinct localities using the ANOVA test. In case of statistical differences of average values, the Tukey test was applied to distinguish group differences, at a 5\% significance level. Linear regression analysis was used to evaluate the relationship between caudex height and species richness. Data analysis was performed as described by Vieira (1980) and Zar (1999) using the SPSS 16.0 programme installed at Universidade Feevale.

\section{Results}

Considering all sites, a total of 31 epiphytic fern species (Table 2) was recorded, representing 16 genera and six families. In species richness, Polypodiaceae (39\%) was followed by Aspleniaceae (22\%), Hymenophyllaceae and Dryopteridaceae (13\%, each). Pteridaceae was represented by three species $(10 \%)$ and Blechnaceae by a single species 


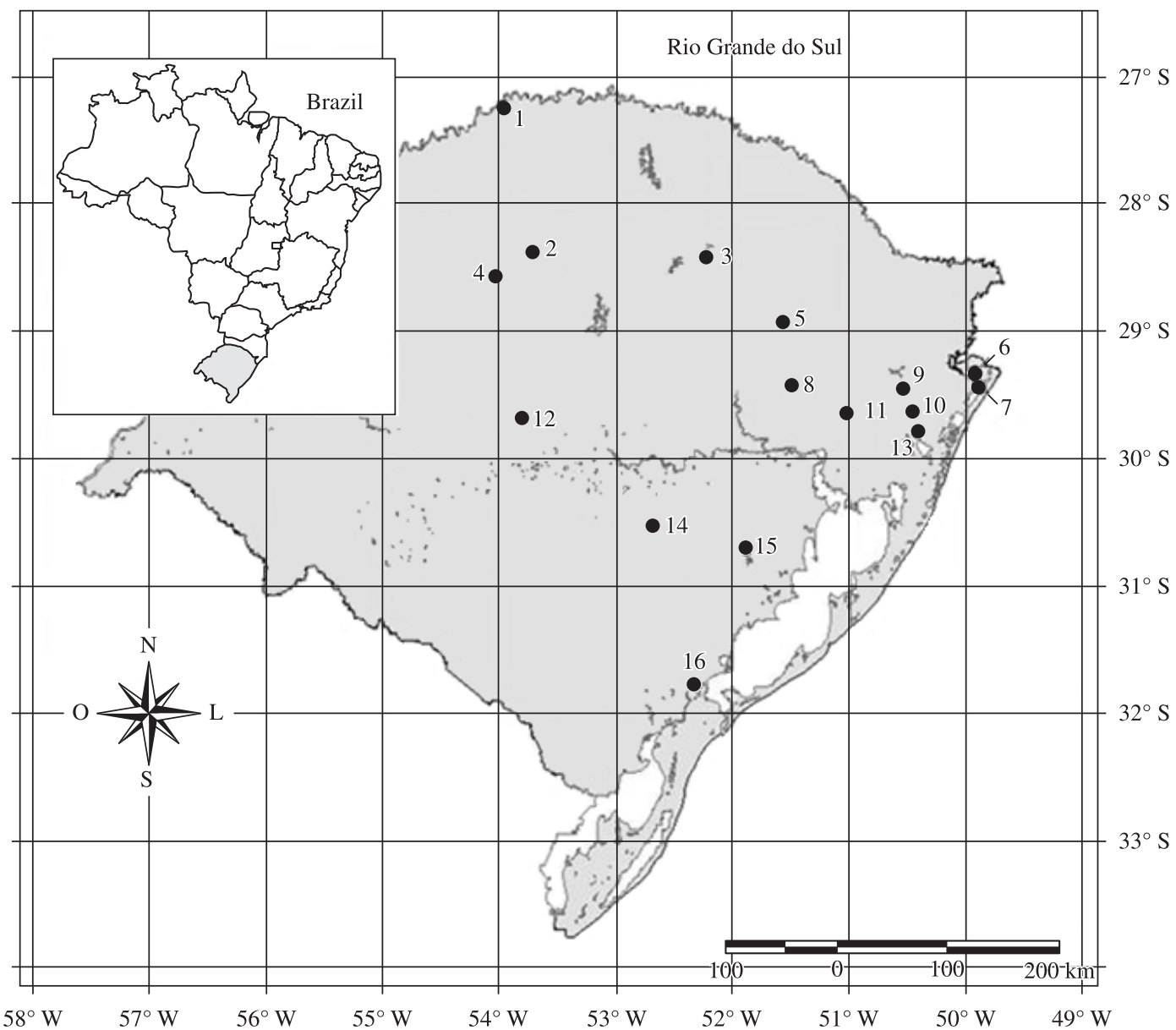

Figure 1. Locations of the 16 study sites in State of Rio Grande do Sul, Brazil. 1 - DeSDF, 2 - BoSDF, 3 - MaMHF, 4 - ApSDF, 5 - VeMHF, 6 - MsDHF, 7 - TcPF, 8 - SsSDF, 9 - SfMHF, 10 - RiSSF, 11 - SaSSF, 12 - SmSDF, 13 - CaDHF, 14 - EsSSF, 15 - CmSSF, 16 - PePF. Acronyms formed according to Table 1.

(3\%). The genus Asplenium L. presented the most species (22\%), followed by Trichomanes L. (13\%).

The highest total specific richness (16 species or 52\%) was recorded for mixed humid forest in São Francisco de Paula (SfMHF) and seasonal semideciduous forest in Sapiranga (SaSSF). The lowest total specific richness (5 or 16\%) was recorded for seasonal deciduous forest in Salvador do Sul (SsSDF). Except for seasonal semideciduous forest in Riozinho (RiSSF), Polypodiaceae presented highest species richness in all sites.

The genera Asplenium and Trichomanes were among those with highest richness in nine study sites. The species presenting generalised occurrence were Trichomanes angustatum Carmich. and Blechnum binervatum (Poir.) C.V.Morton and Lellinger, found in all sites. Campyloneurum nitidum C.Presl. occurred in all sites except in EsSSF, while Vittaria lineata (L.) Sm. and Pecluma pectinatiformis (Lindm.) M.G.Price were not found in four sites (Table 2).

The distribution of the total epiphytic species in different ecological categories (Table 2) showed the dominance of habitual (HAB) holoepiphytes $(61 \%)$, followed by accidental (ACI) holoepiphytes (23\%), facultative (FAC) holoephytes (13\%) and secondary (HMS) hemiepiphytes (3\%). The family presenting only habitual holoepiphytes was Hymenophyllaceae. Polypodiaceae presented the largest number of habitual holoepiphytes. The category of secondary hemiepiphytes is only represented by Blechnum binervatum, which usually germinates on the ground and grows up on the caudex as a hemiepiphyte. However it was also observed germinating directly on the caudex.

At all of the 16 sites, the habitual (HAB) holoepiphytes represented the ecological category with highest specific richness (often $>50 \%$ of the total). At six sites accidental (ACI) holoepiphytes were not found and at five of them this category presented the lowest specific richness. Except for Asplenium claussenii Hieron. and A. inaequilaterale Willd., all accidental holoepiphytes occurred in a single site (Table 2).

Floristic similarity analyses segregated two large groups. The first group (A) was formed only by two geographically 
Table 1. Details of sampling sites. Annual precipitation data were obtained from www.defesacivil.rs.gov.br and vegetation types follow the classification of Teixeira et al. (1986).

\begin{tabular}{lllrll}
\hline \multicolumn{1}{c}{ Location } & Latitude & Longitude & $\begin{array}{c}\text { Altitude } \\
\text { (m) }\end{array}$ & $\begin{array}{c}\text { Annual } \\
\text { precipitation } \\
(\mathbf{m m})\end{array}$ & \multicolumn{1}{c}{$\begin{array}{c}\text { Vegetation } \\
\text { type }\end{array}$} \\
\hline Derrubadas (De) & $27^{\circ} 14^{\prime} \mathrm{S}$ & $53^{\circ} 58^{\prime} \mathrm{W}$ & 388 & 1,635 & Seasonal Deciduous Forest (SDF) \\
Bozano (Bo) & $28^{\circ} 22^{\prime} \mathrm{S}$ & $53^{\circ} 41^{\prime} \mathrm{W}$ & 465 & 1,508 & Seasonal Deciduous Forest (SDF) \\
Marau (Ma) & $28^{\circ} 24^{\prime} \mathrm{S}$ & $52^{\circ} 13^{\prime} \mathrm{W}$ & 571 & 1,435 & Mixed Humid Forest (MHF) \\
Augusto Pestana (Ap) & $28^{\circ} 33^{\prime} \mathrm{S}$ & $54^{\circ} 00^{\prime} \mathrm{W}$ & 452 & 1,531 & Seasonal Deciduous Forest (SDF) \\
Veranópolis (Ve) & $28^{\circ} 55^{\prime} \mathrm{S}$ & $51^{\circ} 33^{\prime} \mathrm{W}$ & 652 & 1,668 & Mixed Humid Forest (MHF) \\
Morrinhos do Sul (Ms) & $29^{\circ} 21^{\prime} \mathrm{S}$ & $49^{\circ} 56^{\prime} \mathrm{W}$ & 80 & 1,682 & Dense Humid Forest (DHF) \\
Três Cachoeiras (Tc) & $29^{\circ} 24^{\prime} \mathrm{S}$ & $49^{\circ} 54^{\prime} \mathrm{W}$ & 15 & 1,535 & Pioneer Formation (PF) \\
Salvador do Sul (Ss) & $29^{\circ} 26^{\prime} \mathrm{S}$ & $51^{\circ} 30^{\prime} \mathrm{W}$ & 493 & 1,476 & Seasonal Deciduous Forest (SDF) \\
São Francisco de Paula (Sf) & $29^{\circ} 27^{\prime} \mathrm{S}$ & $50^{\circ} 33^{\prime} \mathrm{W}$ & 593 & 1,945 & Mixed Humid Forest (MHF) \\
Riozinho (Ri) & $29^{\circ} 38^{\prime} \mathrm{S}$ & $50^{\circ} 27^{\prime} \mathrm{W}$ & 90 & 1,005 & Seasonal Semideciduous Forest (SSF) \\
Sapiranga (Sa) & $29^{\circ} 38^{\prime} \mathrm{S}$ & $51^{\circ} 00^{\prime} \mathrm{W}$ & 570 & 1,131 & Seasonal Semideciduous Forest (SSF) \\
Santa Maria (Sm) & $29^{\circ} 38^{\prime} \mathrm{S}$ & $53^{\circ} 54^{\prime} \mathrm{W}$ & 225 & 1,254 & Seasonal Deciduous Forest (SDF) \\
Caraá (Ca) & $29^{\circ} 42^{\prime} \mathrm{S}$ & $50^{\circ} 17^{\prime} \mathrm{W}$ & 478 & 1,521 & Dense Humid Forest (DHF) \\
Encruzilhada do Sul (Es) & $30^{\circ} 30^{\prime} \mathrm{S}$ & $52^{\circ} 41^{\prime} \mathrm{W}$ & 394 & 1,485 & Seasonal Semideciduous Forest (SSF) \\
Camaquã (Cm) & $30^{\circ} 41^{\prime} \mathrm{S}$ & $51^{\circ} 53^{\prime} \mathrm{W}$ & 192 & 1,667 & Seasonal Semideciduous Forest (SSF) \\
Pelotas (Pe) & $31^{\circ} 46^{\prime} \mathrm{S}$ & $52^{\circ} 26^{\prime} \mathrm{W}$ & 13 & 1,141 & Pioneer Formation (PF) \\
\hline
\end{tabular}

Table 2. Epiphytic ferns on Alsophila setosa Kaulf. at 16 study sites in the State of Rio Grande do Sul, Brazil, and respective ecological categories.

\begin{tabular}{|c|c|c|c|c|c|c|c|c|c|c|c|c|c|c|c|c|c|}
\hline \multirow[t]{2}{*}{ Family/species } & \multirow{2}{*}{$\begin{array}{c}\text { Ecol. } \\
\text { cat. }\end{array}$} & \multicolumn{2}{|c|}{ PF } & \multicolumn{2}{|c|}{ DHF } & \multicolumn{3}{|c|}{ MHF } & \multicolumn{4}{|c|}{ SSF } & \multicolumn{5}{|c|}{ SDF } \\
\hline & & Tc & $\mathbf{P e}$ & Ms & $\mathbf{C a}$ & Ma & Ve & Sf & $\mathbf{R i}$ & $\mathbf{S a}$ & Es & $\mathrm{Cm}$ & De & Bo & Ap & Ss & $\mathrm{Sm}$ \\
\hline \multicolumn{18}{|l|}{ ASPLENIACEAE } \\
\hline $\begin{array}{l}\text { Asplenium claussenii } \\
\text { Hieron. }\end{array}$ & $\mathrm{ACI}$ & & $\mathrm{X}$ & & & $X$ & $\mathrm{X}$ & $\mathrm{X}$ & & & $\mathrm{X}$ & $\mathrm{X}$ & $\mathrm{X}$ & & $\mathrm{X}$ & & \\
\hline Asplenium gastonis Fée & FAC & & & & & & & $\mathrm{X}$ & $\mathrm{X}$ & & & & $\mathrm{X}$ & & & & \\
\hline $\begin{array}{l}\text { Asplenium harpeodes } \\
\text { Kunze }\end{array}$ & HAB & & & & & & & & $\mathrm{X}$ & & & & & & & & \\
\hline $\begin{array}{l}\text { Asplenium inaequilaterale } \\
\text { Willd. }\end{array}$ & ACI & & & & & & & $\mathrm{X}$ & & & & & & & & & $X$ \\
\hline Asplenium incurvatum Fée & HAB & & & & & & & & $\mathrm{X}$ & & & & & & & & \\
\hline $\begin{array}{l}\text { Asplenium mисronatum } \\
\text { C.Presl }\end{array}$ & HAB & $\mathrm{X}$ & & $\mathrm{X}$ & & & & & & $\mathrm{X}$ & & & & & & & \\
\hline $\begin{array}{l}\text { Asplenium scandicinum } \\
\text { Kaulf. }\end{array}$ & HAB & & & $\mathrm{X}$ & $\mathrm{X}$ & $\mathrm{X}$ & & $\mathrm{X}$ & $\mathrm{X}$ & $\mathrm{X}$ & & $\mathrm{X}$ & $\mathrm{X}$ & $\mathrm{X}$ & $\mathrm{X}$ & & \\
\hline \multicolumn{18}{|l|}{ BLECHNACEAE } \\
\hline $\begin{array}{l}\text { Blechnum binervatum } \\
\text { (Poir.) C.V.Morton \& } \\
\text { Lellinger }\end{array}$ & HMS & $\mathrm{X}$ & $\mathrm{X}$ & $\mathrm{X}$ & $\mathrm{X}$ & $\mathrm{X}$ & $\mathrm{X}$ & $\mathrm{X}$ & $\mathrm{X}$ & $\mathrm{X}$ & $\mathrm{X}$ & $\mathrm{X}$ & $\mathrm{X}$ & $\mathrm{X}$ & $\mathrm{X}$ & $\mathrm{X}$ & $\mathrm{X}$ \\
\hline \multicolumn{18}{|l|}{ DRYOPTERIDACEAE } \\
\hline Ctenitis sp.* & ACI & & & & & & & & & & & & & & $\mathrm{X}$ & & \\
\hline $\begin{array}{l}\text { Lastreopsis amplissima } \\
\text { (C.Presl) Tindale }\end{array}$ & $\mathrm{ACI}$ & & & & & & & & & $\mathrm{X}$ & & & & & & & \\
\hline Polystichum sp.* & ACI & & & & & & $\mathrm{X}$ & & & & & & & & & & \\
\hline $\begin{array}{l}\text { Rumohra adiantiformis } \\
\text { (G.Forst.) Ching }\end{array}$ & FAC & $\mathrm{X}$ & $\mathrm{X}$ & & $\mathrm{X}$ & & $\mathrm{X}$ & $\mathrm{X}$ & $\mathrm{X}$ & $\mathrm{X}$ & & $\mathrm{X}$ & & & & & \\
\hline HYMENOPHYLLACEAE & & & & & & & & & & & & & & & & & \\
\hline $\begin{array}{l}\text { Trichomanes anadromum } \\
\text { Rosenst. }\end{array}$ & HAB & & & $\mathrm{X}$ & $\mathrm{X}$ & & & & & & & & & & & & \\
\hline
\end{tabular}

Abbreviation of location and vegetation types according to Table 1. Ecol. cat. = Ecological category; ACI = accidental holoepiphytes; $\mathrm{FAC}=$ facultative holoepiphytes; $\mathrm{HAB}=$ habitual holoepiphytes; HMS = secondary hemiephytes; * sterile plants. 
Table 2. Continued...

\begin{tabular}{|c|c|c|c|c|c|c|c|c|c|c|c|c|c|c|c|c|c|}
\hline \multirow[t]{2}{*}{ Family/species } & \multirow{2}{*}{$\begin{array}{c}\text { Ecol. } \\
\text { cat. }\end{array}$} & \multicolumn{2}{|c|}{ PF } & \multicolumn{2}{|c|}{ DHF } & \multicolumn{3}{|c|}{ MHF } & \multicolumn{4}{|c|}{ SSF } & \multicolumn{5}{|c|}{ SDF } \\
\hline & & Tc & $\mathbf{P e}$ & Ms & $\mathrm{Ca}$ & Ma & Ve & Sf & $\mathbf{R i}$ & $\mathbf{S a}$ & Es & $\mathrm{Cm}$ & De & Bo & Ap & Ss & $\mathrm{Sm}$ \\
\hline $\begin{array}{l}\text { Trichomanes angustatum } \\
\text { Carmich. }\end{array}$ & HAB & $\mathrm{X}$ & $\mathrm{X}$ & $\mathrm{X}$ & $\mathrm{X}$ & $X$ & $\mathrm{X}$ & $\mathrm{X}$ & $\mathrm{X}$ & $\mathrm{X}$ & $\mathrm{X}$ & $\mathrm{X}$ & $\mathrm{X}$ & $X$ & $\mathrm{X}$ & $\mathrm{X}$ & $X$ \\
\hline $\begin{array}{l}\text { Trichomanes polypodioides } \\
\text { L. }\end{array}$ & HAB & $\mathrm{X}$ & & $\mathrm{X}$ & & & & & & & & & & & & & \\
\hline $\begin{array}{l}\text { Trichomanes radicans Sw. } \\
\text { PTERIDACEAE }\end{array}$ & HAB & & & & & & & & & $\mathrm{X}$ & & & & & & & \\
\hline $\begin{array}{l}\text { Adiantum raddianum } \\
\text { C.Presl }\end{array}$ & ACI & & & & & & & $\mathrm{X}$ & & & & & & & & & \\
\hline $\begin{array}{l}\text { Doryopteris pedata (L.) } \\
\text { Fée }\end{array}$ & ACI & & & & & & & & & & & & & & & & $\mathrm{X}$ \\
\hline $\begin{array}{l}\text { Vittaria lineata (L.) Sm. } \\
\text { POLYPODIACEAE }\end{array}$ & HAB & $\mathrm{X}$ & $\mathrm{X}$ & $\mathrm{X}$ & $X$ & & $\mathrm{X}$ & $\mathrm{X}$ & $\mathrm{X}$ & $\mathrm{X}$ & $\mathrm{X}$ & $\mathrm{X}$ & & $\mathrm{X}$ & & $\mathrm{X}$ & \\
\hline $\begin{array}{l}\text { Campyloneurum } \\
\text { austrobrasilianum (Alston) } \\
\text { de la Sota }\end{array}$ & HAB & & & $\mathrm{X}$ & $\mathrm{X}$ & & & $\mathrm{X}$ & & $\mathrm{X}$ & & & & & & & \\
\hline $\begin{array}{l}\text { Campyloneurum nitidum } \\
\text { C.Presl }\end{array}$ & FAC & $\mathrm{X}$ & $\mathrm{X}$ & $\mathrm{X}$ & $X$ & $\mathrm{X}$ & $X$ & $X$ & $\mathrm{X}$ & $\mathrm{X}$ & & $\mathrm{X}$ & $X$ & $\mathrm{X}$ & $X$ & $\mathrm{X}$ & $X$ \\
\hline $\begin{array}{l}\text { Microgramma squamulosa } \\
\text { (Kaulf.) de la Sota }\end{array}$ & HAB & & $\mathrm{X}$ & $\mathrm{X}$ & & & $\mathrm{X}$ & $\mathrm{X}$ & & $\mathrm{X}$ & $\mathrm{X}$ & $\mathrm{X}$ & & & $\mathrm{X}$ & & $\mathrm{X}$ \\
\hline $\begin{array}{l}\text { Microgramma tecta } \\
\text { (Kaulf.) Alston }\end{array}$ & HAB & & & $\mathrm{X}$ & & & & & & & & & & & & & \\
\hline $\begin{array}{l}\text { Microgramma vacciniifolia } \\
\text { (Langsd. \& Fisch.) Copel. }\end{array}$ & HAB & $\mathrm{X}$ & $\mathrm{X}$ & $\mathrm{X}$ & & & & & & & & $\mathrm{X}$ & & & & & \\
\hline $\begin{array}{l}\text { Niphidium rufosquamatum } \\
\text { Lellinger }\end{array}$ & FAC & & & & & & & $\mathrm{X}$ & & $\mathrm{X}$ & & $\mathrm{X}$ & $\mathrm{X}$ & & & & \\
\hline $\begin{array}{l}\text { Pecluma pectinatiformis } \\
\text { (Lindm.) M.G.Price }\end{array}$ & HAB & & $\mathrm{X}$ & & $\mathrm{X}$ & $\mathrm{X}$ & & $\mathrm{X}$ & $\mathrm{X}$ & $\mathrm{X}$ & & $\mathrm{X}$ & $X$ & $\mathrm{X}$ & $X$ & $\mathrm{X}$ & $\mathrm{X}$ \\
\hline $\begin{array}{l}\text { Pecluma recurvata (Kaulf.) } \\
\text { M.G.Price }\end{array}$ & HAB & & & & $\mathrm{X}$ & & & $\mathrm{X}$ & & & & & & & & & \\
\hline $\begin{array}{l}\text { Pecluma truncorum } \\
\text { (Lindm.) M.G.Price }\end{array}$ & НАВ & $\mathrm{X}$ & & $\mathrm{X}$ & $\mathrm{X}$ & & & & & $\mathrm{X}$ & & & & & & & \\
\hline $\begin{array}{l}\text { Pleopeltis angusta Humb. } \\
\text { \& Bonpl. ex Willd. }\end{array}$ & HAB & & $\mathrm{X}$ & & & & & & & $\mathrm{X}$ & & & & & & & \\
\hline $\begin{array}{l}\text { Polypodium catharine } \\
\text { Langsd. \& Fisch. }\end{array}$ & HAB & $\mathrm{X}$ & & $\mathrm{X}$ & & & & & & & $\mathrm{X}$ & $\mathrm{X}$ & & & & & \\
\hline $\begin{array}{l}\text { Polypodium hirsutissimum } \\
\text { Raddi }\end{array}$ & HAB & & & & & & & $\mathrm{X}$ & & $\mathrm{X}$ & & $X$ & & & & & \\
\hline Total & & 10 & 10 & 14 & 1 & 6 & 8 & 16 & 10 & 16 & 6 & 13 & 8 & 6 & 8 & 5 & 7 \\
\hline
\end{tabular}

Abbreviation of location and vegetation types according to Table 1. Ecol. cat. = Ecological category; ACI = accidental holoepiphytes; $\mathrm{FAC}=$ facultative holoepiphytes; HAB = habitual holoepiphytes; HMS = secondary hemiephytes; * sterile plants.

close sites, but with distinct vegetation. The second group (B) included the remaining sites and reflects the natural heterogeneity of the floristic composition in these habitats. Two subgroups can be recognised, one formed only by two close sites presenting different vegetation $(\mathrm{Ba})$, and the other $(\mathrm{Bb})$ where all seasonal deciduous forest sites (SDF) appear together (Cb) (Figure 2).

The average height of the phorophytes in 14 sites was similar. The lower average height was found for seasonal semideciduous forest in Sapiranga (SaSSF), and the highest for seasonal deciduous forest in Santa Maria (SmSDF). The majority of sites (13) presented similar average richness of epiphytes per caudex. In only $25 \%$ of the sites the height of the host plants presented significant relation with specific richness (Table 3 ).

\section{Discussion}

The 31 species on Alsophila setosa represent approximately $10 \%$ of the total of fern species cited by Falavigna (2002) for the State of Rio Grande do Sul. This result shows the importance of this single host species as a microhabitat for epiphytic ferns in the forest environment.

A previous work by Schmitt and Windisch (2005) on the epiphytic ferns in a tract of seasonal semideciduous forest in the State of Rio Grande do Sul reported 14 species on Alsophila setosa. In the present study, the sampled sites within this vegetation revealed 22 species showing low similarity among sites and indicating heterogeneity of the epiphytic fern flora. The total of 31 species indicates that the surrounding environment affects the biodiversity of the 
epiphytic ferns on A. setosa. Habitual holoepiphytes such as Trichomanes anadromum Rosenst., T. polypodioides L., Microgramma tecta (Kaulf.) Alston and Pecluma recurvata (Kaulf.) M.G.Price were found on A. setosa only in dense humid forest, mixed humid forest or pioneer formations. The assembly of several distinct vegetation types presenting heterogenic environmental conditions contributes to the establishment of species with distinct ecological requirements.

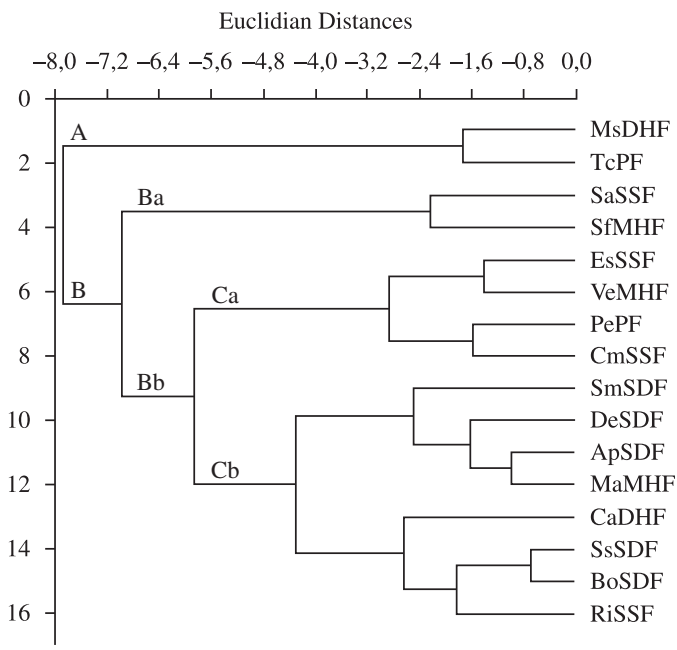

Figure 2. Dendogram of the epiphytic fern communities of the 16 study sites in the State of Rio Grande do Sul, Brazil. Acronyms formed according to Table 1 .
Polypodiaceae, with a larger number of epiphytic species in the study sites (and 39\% of the total) is also the family with the highest richness of epiphytes worldwide (Madison, 1977; Kress, 1986; Benzing, 1990), as well as in the neotropical region (Gentry and Dodson, 1987). The genera Asplenium and Trichomanes, which presented the largest number of species in this study, are considered the richest (more than 100 epiphytic species) on a worldwide basis by Madison (1977) and Kress (1986).

Even in other epiphytic fern surveys, with different sampling methods and distinct phorophyte species, habitual holoepiphytes present highest richness (de la Sota, 1971, 1972; Labiak and Prado, 1998; Schmitt et al., 2005; Fraga et al., 2008). Habitual holoepiphytes present more specialised and diversified adaptations which probably account for their generalised occurrence.

Morphological adaptations of epiphytic species from the Trichomanes genera such as a branched, fine to filiform rhizome, with reduced radicular system and frond size (Dubuisson et al., 2003) contributed to the occurrence of T. angustatum in all studied sites. Other adaptations such as poikilohydry (Benzing, 1987; 1990), fleshy rhizome, nidular habit (Waechter, 1992), trichomes (Müller et al., 1981) and scales, probably account for the generalised occurrence of Campyloneurum nitidum and Pecluma pectinatiformis. The wide distribution of these three species and of Blechnum binervatum and Vittaria lineata suggest that they present high ecological plasticity. On the other hand, although the accidental holoepiphytes were considered the second largest group, they were found (except for two species) on

Table 3. Average phorophyte height and richness of epiphytic ferns on Alsophila setosa Kaulf.; linear regression equations and correlation coefficients $\left(\mathrm{R}^{2}\right)$ from the relation between phorophyte height and richness at 16 study sites in the State of Rio Grande do Sul, Brazil.

\begin{tabular}{|c|c|c|c|c|c|c|}
\hline \multirow[t]{2}{*}{$\begin{array}{l}\text { Study } \\
\text { sites }\end{array}$} & \multicolumn{2}{|c|}{$\begin{array}{c}\text { Phorophyte } \\
\text { height }\end{array}$} & \multicolumn{2}{|c|}{ Richness } & \multicolumn{2}{|c|}{$\begin{array}{c}\text { Parameters of regression } \\
\text { analysis }\end{array}$} \\
\hline & $\begin{array}{c}\text { Average } \pm \text { Sd } \\
(\mathbf{m})\end{array}$ & $\begin{array}{c}\text { Maximum } \\
(\mathrm{m})\end{array}$ & Average \pm Sd & Maximum & $\begin{array}{c}\text { Regression } \\
\text { equation }\end{array}$ & $\mathbf{R}^{2}$ \\
\hline $\mathrm{TcPF}$ & $5.12 \pm 0.98^{\mathrm{abc}}$ & 6.20 & $3.20 \pm 1.03^{\mathrm{abcd}}$ & 5 & $y=-0.0010 x+3.77$ & $0.010 \mathrm{~ns}$ \\
\hline PePF & $4.40 \pm 1.10^{\mathrm{abc}}$ & 5.80 & $4.29 \pm 1.60^{\mathrm{d}}$ & 7 & $y=0.0080 x+0.66$ & $0.320 \mathrm{~ns}$ \\
\hline MsDHF & $4.46 \pm 1.21^{\mathrm{abc}}$ & 6.90 & $1.80 \pm 0.63^{\mathrm{ab}}$ & 3 & $y=-0.0006 x+2.09$ & $0.020 \mathrm{~ns}$ \\
\hline $\mathrm{CaDHF}$ & $3.67 \pm 0.82^{\mathrm{ab}}$ & 5.20 & $3.40 \pm 1.65^{\mathrm{bcd}}$ & 6 & $y=0.0140 x-1.71$ & $0.484 * *$ \\
\hline MaMHF & $4.06 \pm 0.58^{\mathrm{abc}}$ & 4.95 & $3.40 \pm 1.14^{\mathrm{bcd}}$ & 5 & $y=0.0110 x-1.13$ & $0.322 \mathrm{~ns}$ \\
\hline VeMHF & $4.86 \pm 1.08^{\mathrm{abc}}$ & 5.86 & $2.10 \pm 0.74^{\mathrm{abc}}$ & 3 & $y=0.0009 x+1.66$ & $0.020 \mathrm{~ns}$ \\
\hline SfMHF & $4.19 \pm 1.01^{\mathrm{abc}}$ & 5.70 & $4.00 \pm 1.83^{\mathrm{cd}}$ & 6 & $y=0.0140 x-2.03$ & $0.641 * *$ \\
\hline RiSSF & $4.58 \pm 1.21^{\mathrm{abc}}$ & 6.00 & $1.88 \pm 1.13^{\mathrm{ab}}$ & 4 & $y=0.0043 x-0.10$ & $0.215 \mathrm{~ns}$ \\
\hline SaSSF & $3.33 \pm 1.24^{\mathrm{a}}$ & 5.02 & $2.80 \pm 1.23^{\mathrm{abcd}}$ & 5 & $y=0.0073 x+0.35$ & $0.548 *$ \\
\hline EsSSF & $4.09 \pm 1.13^{\mathrm{abc}}$ & 5.32 & $2.20 \pm 1.03^{\mathrm{abc}}$ & 4 & $y=0.0074 x-0.85$ & $0.672 *$ \\
\hline $\mathrm{CmSSF}$ & $4.00 \pm 1.22^{\mathrm{abc}}$ & 5.70 & $2.00 \pm 1.15^{\mathrm{ab}}$ & 4 & $y=0.0058 x-0.33$ & $0.384 \mathrm{~ns}$ \\
\hline DeSDF & $5.56 \pm 2.14^{\mathrm{bc}}$ & 9.00 & $2.40 \pm 0.97^{\mathrm{abcd}}$ & 4 & $y=0.0023 x+1.10$ & $0.271 \mathrm{~ns}$ \\
\hline BoSDF & $5.69 \pm 0.99^{\mathrm{abc}}$ & 8.50 & $2.56 \pm 0.88^{\mathrm{abcd}}$ & 4 & $y=0.0004 x+2.31$ & $0.004 \mathrm{~ns}$ \\
\hline ApSDF & $4.84 \pm 0.99^{\mathrm{abc}}$ & 6.10 & $2.44 \pm 0.73^{\mathrm{abcd}}$ & 4 & $y=0.0035 x+0.73$ & $0.236 \mathrm{~ns}$ \\
\hline SsSDF & $4.57 \pm 0.89^{\mathrm{abc}}$ & 5.50 & $1.40 \pm 0.89^{\mathrm{a}}$ & 3 & $y=0.0058 x-1.25$ & $0.336 \mathrm{~ns}$ \\
\hline SmSDF & $5.93 \pm 1.63^{c}$ & 8.00 & $2.00 \pm 1.07^{\mathrm{ab}}$ & 4 & $y=0.0021 x+0.74$ & $0.106 \mathrm{~ns}$ \\
\hline ANOVA & $\mathrm{P}<0.01$ & & $\mathrm{P}<0.01$ & & & \\
\hline
\end{tabular}

Acronyms of the study sites according to Table 1 . Averages with same letter are same (Tukey test $5 \%$ ). Sd $=\mathrm{Standard}$ deviation $; * \mathrm{P}<0.05 ; * * \mathrm{P}<0.01 ; \mathrm{ns}=$ non-significant. 
a single site in our study, probably due the lack of proper vegetative/biological adaptations.

The distinction between accidental holoepiphytes and facultative ones is not clear cut, and their classification may vary according to the author or local predominant environmental conditions. Rumohra adiantiformis (G.Forst.) Ching, for example, here treated as a facultative epiphyte, was considered by Kersten and Silva (2002) as accidental holoepiphyte in a mixed humid forest in the State of Paraná (Brazil). The classification in ecological categories in the present study is based on the most frequent situation observed in the sampling sites.

The reduced total number of species and the lower average richness observed for seasonal deciduous forest in Salvador do Sul (SsSDF) agree with the observations of Sehnem $(1977 ; 1979)$ that in Southern Brazil the seasonal deciduous forests (SDF) present low fern richness. According to this author, the low specific richness can be due to the lack of connections between the seasonal deciduous forest and dense humid forest.

The floristic similarity analysis demonstrated the heterogeneity of epiphytic ferns in study sites, and indicated that the vegetation type is not the main contributor for this difference. The relevant factors in defining habitats within sites were not evident. Micro-climatic differences were likely to account for much of the different epiphytic assemblages among study areas, and disturbance history, animal interactions and dispersal limitations are also factors that can influence the distribution of species (Roberts et al., 2005).

The affinity between the epiphytes found in mixed humid forest and in the seasonal semideciduous forest (SfMHF and SaSSF) or in the seasonal deciduous forest (MaMHF and ApSDF), confirms the continuity of these forests cited by Rambo (1961), Sehnem (1977) and Marchiori (2004) and the occurrence of some fern species is common as presented by Sehnem $(1977,1979)$, in the State of Rio Grande do Sul. The TcPF site (pioneer formation in Três Cachoeiras) probably has climatic conditions comparable to the MsDHF site (dense humid forest in Morrinhos do Sul) which is located only some $6.5 \mathrm{~km}$ away, on the North coast of the State. This explains the presence of typical dense humid forest (DHF) epiphytic species in pioneer formations (PF) and the great similarity among these two sites.

The present results indicate that once Alsophila setosa is established in the vegetation, the type of forest exerts little influence on the average richness of epiphytes on the caudices. The average richness of similar epiphytic ferns on A. setosa may be related to the fact that space and colonisation time availability are similar, because these host plants also had, in a general way, the same mean average height among the studied sites. Based on the yearly frond production rates and the total frond vestiges on the caudex, Schmitt and Windisch (2006) estimated the age of $4 \mathrm{~m}$ tall Alsophila setosa sporophytes at 28-34 years, in secondary forest formations in the northeastern part of the State of Rio Grande do Sul.

The maximum number of epiphytic fern species found by Heatwole (1993) on Blechnum palmiforme (Thouars) C. Char (5) and by Schmitt et al. (2005) on Dicksonia sellowiana Hook. (6) was lower than the maximum for Alsophila setosa (7). The averages of 3.1 species on B. palmiforme (Heatwole, 1993) and 2.46 species on D. sellowiana (Fraga et al., 2008) are within the range observed on A. setosa. Heatwole (1993) and Schmitt et al. (2005) discovered a correlation between epiphytic species richness and the height of tree ferns, such as occurred in four sites in the present study. Longer caudices (older plants) may present different conditions of microclimate and water supply along their extension, favouring spatial and temporal distribution of the epiphytes. Nieder et al. (1999) indicated that old, structurally diverse trees with many different microhabitats host many different epiphyte species. Yeaton and Galdstone (1982) considered that the period of time during which the substrate is available constitutes a major factor for the colonisation of the phorophytes.

Some of the epiphytic species in this study were observed exclusively or preferentially on tree ferns by previous authors: Asplenium mucronatum C.Presl, A. scandicinum Kaulf., A. harpeodes Kunze, Pecluma truncorum, Trichomanes anadromum, T. angustatum and T. polypodioides (Sehnem, 1977; Waechter, 1992; Cortez, 2001; Sylvestre, 2001). Extraction of adult plants and deforestation are threatening the existence not only of the tree fern species but also of microhabitats for epiphytic fern species, some of them exclusively (or mostly so) found on this kind of substrate. Therefore, additional research on the biology of the tree fern as well as of the epiphytic species is urgently needed as their interactions are still poorly understood.

Acknowledgements - This study was made possible by support and facilities from the Universidade Federal do Rio Grande do Sul - UFRGS (Porto Alegre), Universidade Feevale (Novo Hamburgo) and the Brazilian National Research Council CNPq. Students of Feevale provided welcome help in the project activities. Dr. Joanne Sharpe and Dr. Annette Droste provided welcome suggestions.

\section{References}

BENZING, DH., 1987. Vascular epiphytism: taxonomic participation and adaptative diversity. Annals of the Missouri Botanical Garden, vol. 74, no. 2, p. 183-204.

BENZING, DH., 1990. Vascular epiphytes. Cambridge: Cambridge University Press. 354 p.

BENZING, DH., 1995. The physical mosaic and plant variety in forest canopies. Selbyana, vol. 16, no. 2, p. 159-168.

CORTEZ, L., 2001. Pteridófitas epifitas encontradas en Cyatheaceae y Dicksoniaceae de los bosques nublados de Venezuela. Gayana, vol. 58 , no. 1, p. 13-23.

DUBUISSON, JY., HENNEQUIN, S., RAKOTONDRAINIBE, F. and SCHNEIDER, H., 2003. Ecological diversity and adaptive tendencies in the tropical fern Trichomanes L. (Hymenophyllaceae) with special reference to climbing and epiphytic habits. Botanical Journal of the Linnean Society, vol. 142, no. 1, p. 41-63.

FALAVIGNA, TJ., 2002. Diversidade, formas de vida e distribuição altitudinal das pteridófitas do Parque da Ferradura, Canela (RS), Brasil. São Leopoldo: Universidade do Vale do Rio dos Sinos. [Dissertação de Mestrado] 
FOCHT, T. and PILLAR, VD., 2003. Spatial patterns and relations with site factors in a campos grassland under grazing. Brazilian Journal of Biology, vol. 63, no. 3, p. 423-436.

FRAGA, LL., SILVA, LB. and SCHMITT, JL., 2008. Composição e distribuição vertical de pteridófitas epifíticas sobre Dicksonia sellowiana Hook. (Dicksoniaceae), em floresta ombrófila mista no sul do Brasil. Biota Neotroprica, vol. 8, no. 4, p. 123-129.

GENTRY, AH. and DODSON, CH., 1987. Diversity and biogeography of neotropical vascular epiphytes. Annals of the Missouri Botanical Garden, vol. 74, no. 2, p. 205-233.

HAMMER, Ø., HARPER, DAT. and RYAN, PD., 2001. PAST: Paleontological Statistics: Software Package for Education and Data Analysis. Palaeontologia Electronica, vol. 4, no. 1, 9 p. Available from: http://palaeo-electronica.org/2001_1/past/ issue1_01.htm. Access in: 16/01/2008.

HEATWOLE, H., 1993. Distribution of epiphytes on trunks of the arborescent fern, Blechnum palmiforme, at Gough Island, south Atlantic. Selbyana, vol. 14, p. 46-58.

KERSTEN, RA. and SILVA, SM., 2002. Florística e estrutura do componente epifítico vascular em floresta ombrófila mista aluvial do rio Barigüi, Paraná, Brasil. Revista Brasileira de Botânica, vol. 23 , no. 3, p. 259-267.

KRESS, WJ., 1986. The systematic distribution of vascular epiphytes: an update. Selbyana, vol. 9, no. 1, p. 2-22.

LABIAK, PH. and PRADO, J., 1998. Pteridófitas epífitas da Reserva Volta Velha, Itapoá, Santa Catarina, Brasil. Boletim do Instituto de Botânica, vol. 11, p. 1-79.

MADISON, M., 1977. Vascular epiphytes: their systematic occurrence and salient features. Selbyana, vol. 2, no. 1, p. 1-13.

MARCHIORI, JNC., 2004. Fitogeografia do Rio Grande do Sul: campos sulinos. Porto Alegre: EST.

MEDEIROS, AC., LOOPE, LL. and ANDERSON, SJ., 1993. Differential colonization by epiphytes on native (Cibotium spp.) and alien (Cyathea cooperi) tree ferns in a Hawaiian rain forest. Selbyana, vol. 14, p. 71-74.

MORAN, RC., KLIMAS, S. and CARLSEN, M., 2003. Lowtrunk epiphytic ferns on tree ferns versus angiosperms in Costa Rica. Biotropica, vol. 35, no. 1, p. 48-56.

MORENO, JA., 1961. Clima do Rio Grande do Sul. Porto Alegre: Secretaria da Agricultura.

MUCUNGUZI, P., 2007. Diversity and distribution of vascular epiphytes in the forest lower canopy in Kibale National Park, western Uganda. African Journal of Ecology, vol. 45, suppl. 3, p. $120-125$.

MÜLLER, L., STARNECKER, G. and WINKLER, S., 1981. Zur ökologie epiphytisher farne in Südbrasilien. I. Saugschuppen. Flora, vol. 171, p. 55-63.

NIEDER, J., IBISCH, PL. and BARTHLOTT, W., 1997. Biodiversidad de epífitas: una cuestión de escala. Revista del Jardín Botánico Nacional, vol. 17/18, p. 59-62.

NIEDER, J., ENGWALD, S. and BARTHLOTT, W., 1999. Patterns of neotropical epiphyte diversity. Selbyana, vol. 20, no. 1, p. 66-75.

PAGE, CN., 2002. Ecological strategies in fern evolution: a neopteridological overview. Review of Palaeobotany and Palynology, vol. 119 , no. 1 , p. 1-33.

QUADROS, FLF. and PILLAR, VP., 2002. Transições florestacampo no Rio Grande do Sul. Revista Ciência e Ambiente, vol. 24, p. 109-118.
RAMBO, B., 1961. Migration routes of the south Brazilian rain forest. Pesquisas, Série Botânica, vol. 12, p. 1-54.

ROBERTS, NR., DALTON, PJ. and JORDAN, GJ., 2005. Epiphytic ferns and bryophytes of Tasmanian tree-ferns: a comparison of diversity and composition between two host species. Austral Ecology, vol. 30, no. 2, p. 146-154.

RUDOLPH, D., RAUER, G., NEIDER, J. and BARTHLOTT, W., 1998. Distributional patterns of epiphytes in the canopy and phorophyte characteristics in a western Andean rain forest in Ecuador. Selbyana, vol. 19, no. 1, p. 27-33.

SCHMITT, JL. and WINDISCH, PG., 2005. Aspectos ecológicos de Alsophila setosa Kaulf. (Cyatheaceae, Pteridophyta) no sul do Brasil. Acta Botanica Brasilica, vol. 19, no. 4, p. 861-867.

SCHMITT, JL. and WINDISCH, PG., 2006. Growth rates and age estimates of Alsophila setosa Kaulf in Southern Brazil. American Fern Journal, vol. 96, no. 4, p. 103-111.

SCHMITT, JL., BUDKE, JC. and WINDISCH, PG., 2005. Aspectos florísticos e ecológicos de pteridófitas epifíticas em cáudices de Dicksonia sellowiana Hook. (Pteridophyta, Dicksoniaceae), São Francisco de Paula, RS, Brasil. Pesquisas, Série Botânica, vol. 56 , no. 1 , p. 161-172.

SEHNEM, A., 1977. As filicíneas do sul do Brasil, sua distribuição geográfica, sua ecologia e suas rotas de migração. Pesquisas, Série Botânica, vol. 31, p. 1-108.

SEHNEM, A., 1979. Semelhanças e diferenças nas formações florestais do sul do Brasil. Acta Biologica Leopoldensia, vol. 1, no. 1 , p. 111-135.

SMITH, AR., KATHLEEN, MP., SCHUETTPELZ, E., KORALL, P., SCHNEIDER, H. and WOLF, PG., 2006. A classification for extant ferns. Taxon, vol. 55, no. 3, p. 705-731.

SOTA, ER., 1971. El epifitismo y las pteridofitas en Costa Rica (America Central). Nova Hedwigia, vol. 21, no. 2-4, p. 401-465.

SOTA, ER., 1972. Las pteridofitas y el epifitismo en el Departamento del Choco (Colombia). Anales de la Sociedad Cientifica Argentina, vol. 31 , no. $5-6$, p. $245-278$

SYLVESTRE, LS., 2001. Revisão taxonômica das espécies da Família Aspleniaceae A. B. Frank ocorrentes no Brasil. São Paulo: Universidade de São Paulo. [Tese de Doutorado]

TEIXEIRA, MB., COURA NETO, AB., PASTORE, U. and RANGEL FILHO, ALR., 1986. Vegetação. In Levantamento de recursos naturais. Rio de Janeiro: Instituto Brasileiro de Geografia e Estatística - IBGE. p. 541-620. (vol. 33)

VIEIRA, S., 1980. Introdução à bioestatística. 3 ed. Rio de Janeiro: Campus.

WAECHTER, JL., 1992. O epifitismo vascular na Planície costeira do Rio Grande do Sul. São Carlos: Universidade Federal de São Carlos. [Tese de Doutorado]

WINDISCH, PG., 2002. Fern conservation in Brazil. Fern Gazette, vol. 16 , no. 6-8, p. 295-300

YEATON, RI. and GLADSTONE, DE., 1982. The pattern of colonization of epiphytes on Calabash Trees (Crescentia alata HBK.) in Guanacaste Province, Costa Rica. Biotropica, vol. 14, no. 2, p. 137-140.

ZAR, JH., 1999. Biostatistical analysis. 4 ed. New Jersey: Prentice-Hall. 